\title{
Neutral atoms in ionic lattices: Stability and ground-state properties of $\mathrm{KCl}: \mathrm{Ag}^{0}$
}

\author{
J. A. Aramburu, M. Moreno, * and I. Cabria \\ Departamento de Ciencias de la Tierra y Física de la Materia Condensada, Facultad de Ciencias, Universidad de Cantabria, \\ 39005 Santander, Spain \\ M. T. Barriuso \\ Departamento de Física Moderna, Facultad de Ciencias, Universidad de Cantabria, 39005 Santander, Spain \\ C. Sousa, C. de Graaf, and F. Illas \\ Departament de Química Física i Centre Especial de Recerca en Química Teòrica, Universitat de Barcelona, E-08028 Barcelona, Spain
}

(Received 17 December 1999; revised manuscript received 17 April 2000)

\begin{abstract}
The equilibrium geometry of $\mathrm{Ag}^{0}$ centers formed at cation sites in $\mathrm{KCl}$ has been investigated by means of total-energy calculations carried out on clusters of different sizes. Two distinct methods have been employed: First, an ab initio wave-function based method on embedded clusters and second, density-functional theory (DFT) methods on clusters in vacuo involving up to 117 atoms. In the $a b$ initio calculations the obtained equilibrium $\mathrm{Ag}^{0}-\mathrm{Cl}^{-}$distance $R_{e}$ is $3.70 \AA$, implying a large outward relaxation of $18 \%$, along with $7 \%$ relaxation for the distance between $\mathrm{Ag}^{0}$ and the first $\mathrm{K}^{+}$ions in $\langle 100\rangle$ directions. A very similar result is reached through DFT with a 39-atom cluster. Both approaches lead to a rather shallow minimum of the total-energy surface, the associated force constant of the $A_{1 g}$ mode is several times smaller than that found for other impurities in halides. These conclusions are shown to be compatible with available experimental results. The shallow minimum is not clearly seen in DFT calculations with larger clusters. The unpaired electron density on silver and $\mathrm{Cl}$ ligands has been calculated as function of the metal-ligand distance and has been compared with values derived from electron-paramagnetic resonance data. The DFT calculations for all cluster sizes indicate that the experimental hyperfine and superhyperfine constants are compatible when $R_{e}$ is close to $3.70 \AA$. The important relation between the electronic stability of a neutral atom inside an ionic lattice and the local relaxation is established through a simple electrostatic model. As most remarkable features it is shown that (i) the cationic $\mathrm{Ag}^{0}$ center is not likely to be formed inside $\mathrm{AgCl}$, (ii) in the $\mathrm{Ag}^{0}$ center encountered in $\mathrm{SrCl}_{2}$, the silver atom is probably located at an anion site, and (iii) the properties of a center-like $\mathrm{KCl}: \mathrm{Ag}^{0}$ would experience significant changes under hydrostatic pressures of the order of $6 \mathrm{GPa}$.
\end{abstract}

\section{INTRODUCTION}

Doped insulating and semiconductor materials are widely investigated due to the new optical, magnetic, and electrical properties introduced by a given impurity in a crystal lattice. Usually the oxidation state of isolated impurities corresponds to that found for the same element in pure inorganic compounds. However, apart from more common impurities like $\mathrm{Tl}^{+}$or $\mathrm{Ni}^{2+}$, in some crystal lattices it is also possible to stabilize isolated impurities like $\mathrm{Tl}^{2+}$ or $\mathrm{Ni}^{+}$, which are not usual constituents of inorganic compounds. ${ }^{1}$ Such unstable impurities are formed inside a given host lattice by the action of ultraviolet or $x$ irradiation and the subsequent trapping of the released electrons or holes. One can expect that if the released electron is not delocalized in the conduction band but bound to a $\mathrm{M}^{n+}$ cation, the formation of the $\mathrm{M}^{(n-1)+}$ species involves a significant local lattice relaxation. Knowledge of such a relaxation is certainly a prerequisite for understanding all the associated physicochemical properties due to the unstable $\mathrm{M}^{(n-1)+}$ species and, in particular, its electronic stability.

The determination of the actual equilibrium distance $R_{e}$ between the unstable $\mathrm{M}^{(n-1)+}$ species and its nearest neighbors (ligands) is, however, not a simple task. In fact, aside from the nonexistence of pure compounds involving the
$\mathrm{M}^{(n-1)+}$ oxidation state, the extended $\mathrm{x}$-ray-absorption fine structure (EXAFS) techniques can hardly be applied for solving these problems. The stable $\mathrm{M}^{n+}$ and the unstable $\mathrm{M}^{(n-1)+}$ species are usually both present in a given sample and the EXAFS spectrum of both species would appear around the same $x$-ray absorption energy. Moreover a minimum impurity concentration lying between $0.1 \%$ and $3 \%$ is required to detect the EXAFS spectrum of an impurity. ${ }^{2}$ Therefore, the use of theoretical calculations can be very helpful to clarify the $R_{e}$ value for unstable species. Not only can one determine $R_{e}$ from the dependence of the total energy on the impurity-ligand distance $R$, but also find which is, among all optical or electron-paramagnetic resonance (EPR) parameters, the most sensitive one to changes of $R$. By analyzing the experimental value of that parameter for different lattices, useful information about $R_{e}$ and its variations along a series can be obtained. This procedure has been employed in cases like $\mathrm{Tl}^{2+}$ or $\mathrm{Ni}^{+}$in different insulating lattices, ${ }^{3,4}$ as well as for stable impurities like $\mathrm{Mn}^{2+}, \mathrm{Cr}^{3+}$, $\mathrm{Cu}^{2+}, \mathrm{Ni}^{2+}$, or $\mathrm{Fe}^{3+} \cdot{ }^{5-11}$

Among unstable species originated after electron trapping, the study of neutral silver atoms at cationic positions in alkali halides is especially attractive. The formation of such neutral species has been proven by means of EPR and electron-nuclear double resonance techniques, ${ }^{12-16}$ their 
main properties have been reviewed by Nistor, Shoemaker, and Ursu. ${ }^{17}$ The experimental hyperfine constant $A$ of $\mathrm{KCl}: \mathrm{Ag}^{0}$ is practically equal to that for the free silver atom $A_{0} \cdot{ }^{12,14,16}$ In particular, $A$ is found to be only about $5 \%$ smaller than $A_{0}$, which is $1718 \mathrm{MHz}$ for the ${ }^{107} \mathrm{Ag}$ isotope.

Apart from understanding the conditions for stabilizing $\mathrm{Ag}^{0}$ in a lattice like $\mathrm{KCl}$, such species display a rich opticalabsorption spectrum ${ }^{12,18-21}$ that needs to be explained in detail. For instance, the first absorption maximum at $2.92 \mathrm{eV}$ in $\mathrm{KC}: \mathrm{Ag}^{0}$ has been assigned to come from the $5 s \rightarrow 5 p$ transition of free $\mathrm{Ag}^{0} \cdot{ }^{12,18}$ Nevertheless photoconductivity measurements indicate that the nonrelaxed $5 p$ level is placed within the conduction band of $\mathrm{KCl}$, and hence, it cannot have a localized character. ${ }^{12,21}$ Aside from this band, four more optical bands are due to $\mathrm{Ag}^{0}$ placed in $\mathrm{KCl}^{19,20}$ The most intense of such transitions (appearing at $6.3 \mathrm{eV}$ for $\mathrm{KCl}: \mathrm{Ag}^{0}$ ) was tentatively assigned to be a ligand to metal charge transfer (CT) transition. ${ }^{22}$ This assignment is somewhat surprising, as when iso-electronic cations are considered, the energy of CT transitions decreases as far as the nominal charge increases if the ligand is kept. ${ }^{23}$ Following this trend a much higher energy for the charge transfer excitation is expected for a neutral impurity.

A first analysis of the isotropic superhyperfine constant $A_{s}$ in $\mathrm{KCl}: \mathrm{Ag}^{0}$ indicated ${ }^{24,25}$ that $R_{e}$ is significantly larger than the $\mathrm{K}^{+}-\mathrm{Cl}^{-}$distance of the perfect host lattice $R_{0}$ $=3.145 \AA$. In particular, such analysis suggested that if the $\mathrm{Ag}^{+}-\mathrm{Cl}^{-}$distance in $\mathrm{KCl}: \mathrm{Ag}^{+}$is around $2.8 \AA$, it becomes close to $3.7 \AA$ after electron trapping. A similar conclusion was obtained in the case of other alkali halides containing the $\mathrm{Ag}^{0}$ center. ${ }^{25}$

Up to now no attempts have been undertaken to determine the proper $R_{e}$ value in $\mathrm{KCl}: \mathrm{Ag}^{0}$ through total-energy calculations as a function of $R$. Self-consistent charge extended Hückel (SCCEH) and multiple scattering $\mathrm{X} \alpha(\mathrm{MS}-\mathrm{X} \alpha)$ calculations on $\mathrm{KCl}: \mathrm{Ag}^{0}$ at different $R$ values have been carried out in order to explain the experimental optical and EPR parameters due to the silver center in $\mathrm{KCl}^{26}$ These calculations showed that the experimental spectroscopic parameters are better explained assuming a significant outward relaxation around the silver atom.

The proper value of the local relaxation in $\mathrm{KCl}: \mathrm{Ag}^{0}$ is however not definitely settled. After some preliminary studies $^{27}$ in which the relaxation was only qualitatively considered, different estimates have been proposed in the literature. From the analysis of experimental quadrupole tensor, Holmberg, Unruh, and Friauf ${ }^{16}$ suggest an outward relaxation of $10 \%$ when $\mathrm{K}^{+}$is replaced by $\mathrm{Ag}^{0}$. Bucher ${ }^{28}$ proposes, however, a lower value $(4 \%)$ assuming that the relaxation in $\mathrm{KCl}: \mathrm{Ag}^{0}$ essentially coincides with that for the cation vacancy. However, this conclusion is hardly compatible with the $14 \%$ relaxation calculated by Veliah, Pandey, and Marshall ${ }^{29}$ on $\mathrm{KF}: \mathrm{Ag}^{0}$.

The present paper is aimed to determine the proper relaxation around $\mathrm{Ag}^{0}$ in $\mathrm{KCl}$ by means of theoretical calculations on clusters of different size centered at $\mathrm{Ag}^{0}$. For this purpose, we compute the ground-state energy of the clusters for several local relaxations using two distinct methods. One of them is founded on the density-functional theory (DFT), ${ }^{30-33}$ and the other on the complete active space self-consistent field followed by complete active space second-order pertur- bation theory methodology $\mathrm{y}^{34,35}$ implemented in the MOLCAS4 package. $^{36}$ Accurate results on distances have been obtained by both kind of methods. ${ }^{37,38}$ More approximate methods like MS-X $\alpha$ are not useful for this purpose albeit they reproduce reasonably well the electronic properties at the experimental $R_{e}$ value. ${ }^{3,4}$ Clusters have been considered that take into account geometrical relaxation up to the second neighbors in the $\langle 100\rangle$ direction. As expected from the theory of elasticity, the relaxation effects on the first $\mathrm{K}^{+}$ions in $\langle 100\rangle$ directions are not negligible for a relaxation of about $20 \%$ of the first shell. More details on the calculations are given in Sec. II.

To obtain additional information on the equilibrium geometry of $\mathrm{KCl}: \mathrm{Ag}^{0}$ attention is also focused on the unpaired spin density on the silver atom $\left(f_{\mathrm{Ag}}\right)$ as well as the contributions to the unpaired spin density of the $3 p_{\sigma}\left(f_{\sigma}\right)$ and the $3 s\left(f_{s}\right)$ orbitals of chlorine. ${ }^{39}$ The first density is related to the hyperfine constant $A$, while the other two are related to the superhyperfine tensor of the ground state of the silver center. The $f_{s}$ density (directly related to the isotropic superhyperfine constant $A_{s}$ ) has been shown ${ }^{3,5}$ to be highly dependent upon $R$ for octahedral transition-metal complexes involving unpaired $\sigma$ electrons. Microscopically $A_{s}$ is directly related to the unpaired spin density on a $3 s(\mathrm{Cl})$ orbital through the expression ${ }^{24}$

$$
A_{s}(\mathrm{MHz})=4250 f_{s} .
$$

The comparison made in Sec. III between $f_{s}=0.88 \%$ (derived from the experimental $A_{s}=36.3 \mathrm{MHz}$ ) and the calculated one using different clusters, supports that the $R_{e}$ value for $\mathrm{Ag}^{0}$ in $\mathrm{KCl}$ indeed lies around $3.7 \AA$. Also the analysis of the hyperfine constant $A$, sheds an important light on the conditions for observing an unpaired electron highly localized on the silver atom.

In order to better characterize the ground state of $\mathrm{Ag}^{0}$ in $\mathrm{KCl}$, the frequency of the symmetric mode $\omega_{A}$ of the $\mathrm{AgCl}_{6}$ complex embedded in the $\mathrm{KCl}$ lattice, has also been calculated. It turns out that the energy minimum is rather shallow, the associated force constant is about five times smaller than that corresponding to the $\mathrm{CrCl}_{6}{ }^{3-}$ complex. ${ }^{40}$ This point is also discussed in detail in Sec. V.

Another important question concerns the formation of $\mathrm{Ag}^{0}$ in other lattices. For instance, in $\mathrm{AgCl}$, the trapped electrons do not give rise to $\mathrm{Ag}^{0}$ but to a shallow donor center whose nature is still not fully clarified. ${ }^{41-43}$ A similar situation is encountered in $\mathrm{Na}_{1-x} \mathrm{Ag}_{x} \mathrm{Cl}$ mixed crystals when the $x$ fraction is higher than $30 \%{ }^{44}$ Particular attention is addressed upon the relation between the local relaxation and the stability of a neutral silver atom inside an ionic lattice. Using a simple model, the condition of electronic stability of $\mathrm{Ag}^{0}$ at cationic positions in $\mathrm{KCl}$ and in other lattices like $\mathrm{AgCl}$ or $\mathrm{SrCl}_{2}$ is discussed. In the light of this simple model, the possible effects on the ground state properties of the $\mathrm{KCl}: \mathrm{Ag}^{0}$ system under hydrostatic pressure are also analyzed.

\section{COMPUTATIONAL INFORMATION}

\section{A. ROHF calculations}

To determine the relaxation around the $\mathrm{Ag}^{0}$ impurity, we construct a cluster containing the silver impurity and its first 


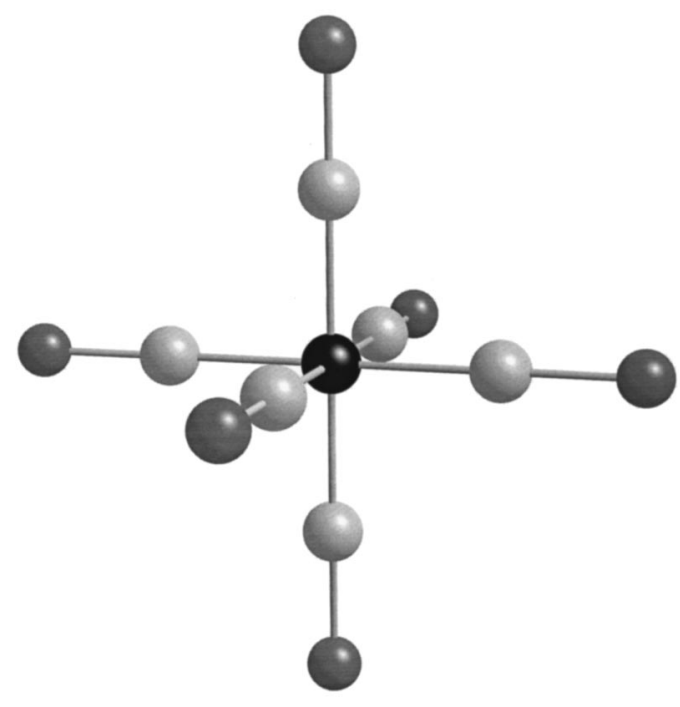

FIG. 1. $\mathrm{AgCl}_{6} \mathrm{~K}_{6}$ cluster model used in ROHF geometry optimization calculations. The black sphere represents the $\mathrm{Ag}^{0}$ impurity, dark gray spheres the $\mathrm{K}$ ions, and light spheres model the $\mathrm{Cl}$ ions. The embedding of the cluster is not depicted.

and second neighbors in the $\langle 100\rangle$ direction, i.e., a $\mathrm{AgCl}_{6} \mathrm{~K}_{6}$ cluster in which all atoms are allowed to relax their position as a reaction on the presence of the $\mathrm{Ag}^{0}$ impurity (Fig. 1). These $\mathrm{Cl}^{-}$and $\mathrm{K}^{+}$ions will be referred as $\mathrm{Cl}^{(1)}$ and $\mathrm{K}^{(4)}$, respectively, as they correspond to the first and fourth atomic shell around $\mathrm{Ag}^{0}$. Hereafter, the distance between $\mathrm{Ag}^{0}$ and $\mathrm{K}^{(4)}$ will be denoted as $R^{(4)}$. The equilibrium $\mathrm{Ag}-\mathrm{Cl}^{(1)}$ distance $R_{e}$ is determined as the minimum of the restricted open shell Hartree-Fock (ROHF) potential-energy surface of the ${ }^{2} A_{1 g}$ ground state. Valence electrons are described with a Gaussian-type basis set, whereas the core electrons are represented by a Cowan-Griffin effective core potential (ECP). These ECP's reduce the size of the calculation somewhat and moreover account for the scalar relativistic effects, which are expected to be important for $\mathrm{Ag}$. To describe the $\mathrm{Ag}^{0}$ valence electrons $\left(4 p^{6}, 4 d^{10}, 5 s^{1}\right)$, we apply a $[11 s, 8 p$, $7 d] /(4 s, 4 p, 4 d)$ basis set and for the $\mathrm{Cl}^{-}$and $\mathrm{K}^{+}$valence electrons $\left(3 s^{2}, 3 p^{6}\right.$, and $3 p^{6}$, respectively) we use a $[7 s$, $7 p] /(3 s, 3 p)$ basis set. ${ }^{45,46}$

In order to obtain accurate estimates of the interatomic distances in the cluster, it is essential to account for the shortrange interaction due to Pauli repulsion supplemented by Coulomb and exchange effects brought about by the charge distributions of the neighboring centers. Here, we include these effects by embedding the cluster in several shells of $a b$ initio embedding model potentials (AIEMP), ${ }^{47-49}$ which are derived for the pure $\mathrm{KCl}$ crystal as described in Ref. 49. These potentials are used to represent all remaining atoms in a cube running from $-a$ to $a$ (being $a$ the lattice parameter of the pure $\mathrm{KCl}$ crystal) extended with the $\mathrm{K}^{+}$ions at $(3 / 2$, $1 / 2,0)$ and the $\mathrm{Cl}^{-}$ions at $(3 / 2,0,0)$ and $(3 / 2,1 / 2,1 / 2)$. Following the conclusions of Ref. 50, the six AIEMP's representing the $\mathrm{Cl}^{-}$ions at $(3 / 2,0,0)$ are provided with a $(1 s, 1 p)$ basis set to maintain the strong orthogonality between cluster and surroundings. In addition, the long-range electrostatic interaction of the cluster with the rest of the crystal is incorpo- rated in the material model by an array of Evjen point charges to complete a cube of $4 a$ length around the central atom.

The accuracy we can expect when predicting the $\mathrm{Ag}-\mathrm{Cl}$ bond length with the present material model, basis sets, and $\mathrm{N}$-electron wave-function approximation can be assessed by calculating the $\mathrm{K}^{+}-\mathrm{Cl}^{-}$distance for the pure $\mathrm{KCl}$ crystal. For this purpose, we construct a $\mathrm{KCl}_{6}{ }^{5-}$ cluster embedded in a similar environment as the $\mathrm{AgCl}_{6} \mathrm{~K}_{6}$ cluster mentioned above. A Hartree-Fock calculation for the ${ }^{1} A_{1 g}$ ground state gives a bond distance of $3.174 \AA$, which is only $0.029 \AA$ longer than the experimental distance. A further improvement can be obtained by accounting for electron correlation effects and/or improving the basis sets, but these are only minor changes and do not change the qualitative picture of the Hartree-Fock calculation. The MP2 bond length is 3.161 $\AA$ with the present basis sets and after adding an extra $p$ and $d$ function to the $\mathrm{Cl}$ basis, the bond length turns out to be 3.168 and $3.135 \AA$ for Hartree-Fock and MP2, respectively. Hence, we conclude that Hartree-Fock calculations with the present choice of basis sets, cluster size, and embedding give a very acceptable estimate of the experimental lattice parameter of pure $\mathrm{KCl}$ and hence, calculations are also expected to give a reasonable estimate of the $\mathrm{Ag}^{0}-\mathrm{Cl}$ bond length.

\section{B. Density-functional calculations}

Most of the calculations of this paper are based on DFT and have been performed using the Amsterdam density functional (ADF) code. ${ }^{31,32}$ Clusters in vacuo, centered at the silver atom including up to a total of 117 atoms, have been used. Only the results of the most relevant clusters containing 39 and 81 atoms (depicted in Fig. 2) are discussed here. The 39-atom cluster $\mathrm{AgCl}_{6} \mathrm{~K}_{12} \mathrm{Cl}_{8} \mathrm{~K}_{6} \mathrm{Cl}_{6}{ }^{2-}$ includes $\mathrm{Cl}^{(1)}$, $\mathrm{K}^{(2)}, \mathrm{Cl}^{(3)}, \mathrm{K}^{(4)}$, and $\mathrm{Cl}^{(9)}$ atoms and hence has three atoms in the $\langle 100\rangle$ directions. The 81-atom cluster $\mathrm{AgCl}_{6} \mathrm{~K}_{12} \mathrm{Cl}_{8} \mathrm{~K}_{6} \mathrm{Cl}_{24} \mathrm{~K}_{24}{ }^{4+}$ involves the six first shells of atoms around $\mathrm{Ag}^{0}$. Triple zeta basis functions of quality IV, which are implemented in the ADF code, are employed. For $\mathrm{Ag}$, electrons up to the $4 p$ shell are kept frozen while for $\mathrm{Cl}$ and $\mathrm{K}$ electrons up to the $2 p$ and $3 p$ shell, respectively, are included in the core. The local density approximation (LDA) exchange-correlation energy is computed according to Vosko, Wilk, and Nusair's parameterization of electron gas data. ${ }^{51}$ For the generalized gradient approximation (GGA) calculations, we opted for the Becke-Perdew functional, which uses Becke's gradient correction ${ }^{52}$ to the local expression of the exchange energy and Perdew's gradient correction $^{53}$ to the local expression of the correlation energy. The equilibrium distances obtained through LDA and GGA schemes differ by less than $1 \%$ for clusters where a minimum in the total energy is found. Such a difference is quite comparable to that obtained studying $\mathrm{Cr}^{3+}$ impurities in fluoroelpasolites through clusters that include up to the third neighbors of the impurity. ${ }^{33}$ In the remainder of the paper, we only list the DFT results obtained with the GGA functional of Becke and Perdew.

For the 39-atom cluster where the second and third ions along $\langle 100\rangle$ directions $\left(\mathrm{K}^{(4)}\right.$ and $\mathrm{Cl}^{(9)}$, respectively) are included, two types of calculations have been performed. In the first place we keep all the atoms in the cluster fixed 

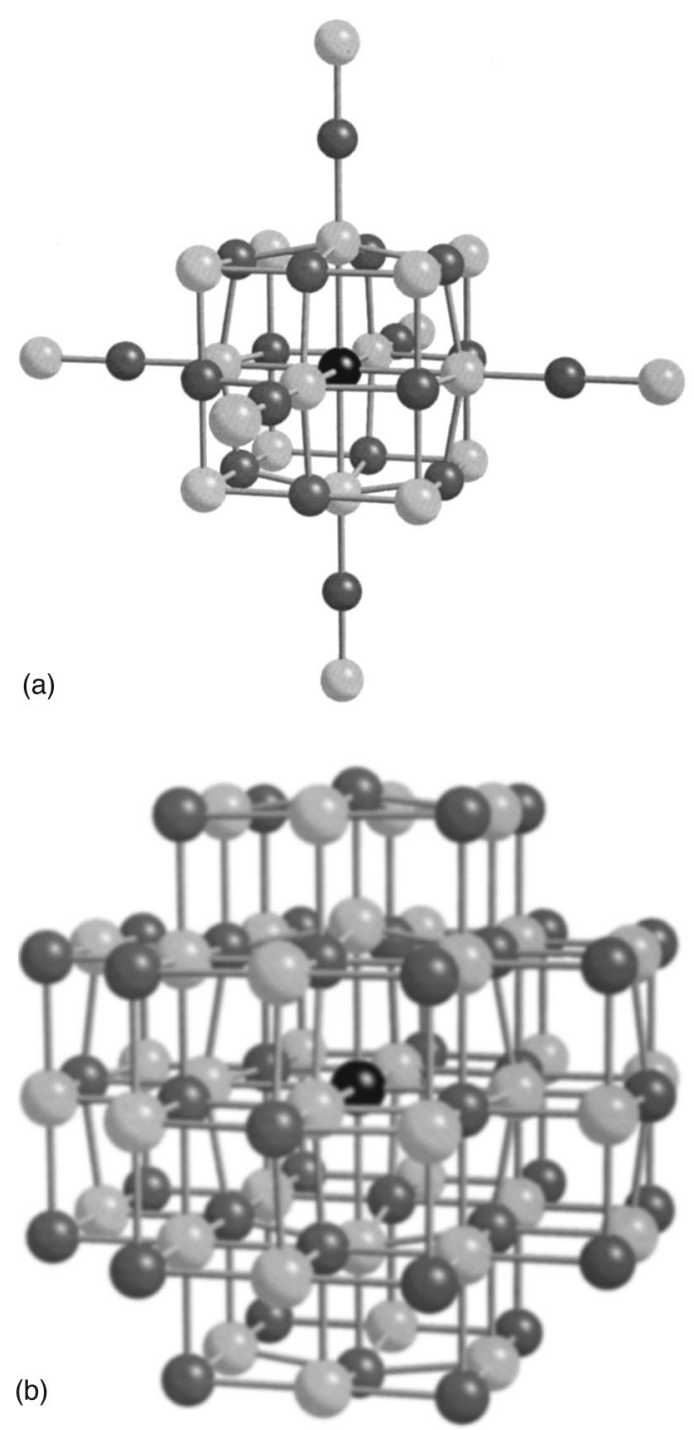

FIG. 2. The 39- and the 81-atom cluster models used in the DFT calculations. The black sphere represents the $\mathrm{Ag}^{0}$ impurity, dark gray spheres the $\mathrm{K}$ ions, and light spheres depict the $\mathrm{Cl}$ ions.

except for the ligands $\mathrm{Cl}^{(1)}$, and secondly, the relaxation of $\mathrm{K}^{(4)}$ ions along $\langle 100\rangle$ directions is also taken into account.

To test the reliability of the DFT calculations, the $\mathrm{KCl}$ lattice has been simulated by means of different clusters centered at a $\mathrm{K}^{+}$ion. If only the distance $R$, to the first $\mathrm{Cl}^{-}$ neighbors is taken as variable the equilibrium distance is found to be $3.19 \AA$ for a cluster involving 27 atoms and 3.12 $\AA$ when the number of atoms is equal to 81 . This indicates that also the DFT based methods reproduce the equilibrium geometry of the host lattice with satisfactory accuracy.

The $R$ dependence of $f_{s}$ has not only been explored by DFT calculations but also by means of MS X $\alpha$ calculations on an 81-atom cluster. In this method the election of the atomic sphere radii is crucial to obtain reliable results. This choice is even more important in this system where very large relaxations are expected. In agreement with our experience in previous calculations, adequate values of atomic sphere radii were chosen in order to achieve an overlap between neighbor spheres of $25 \%$. More details can be found in Ref. 26.

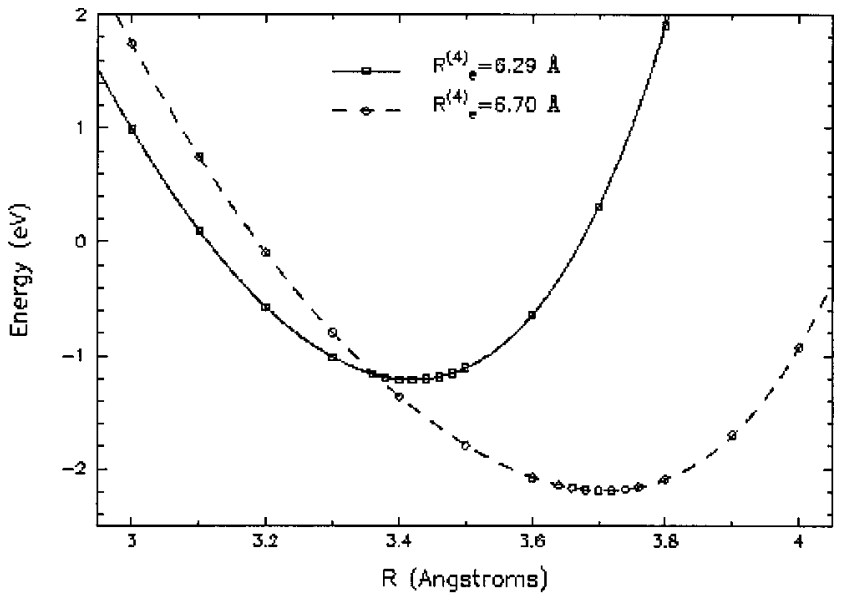

FIG. 3. Results obtained in ROHF calculations for the $R$ (in $\AA$ ) dependence of the energy (in eV) of the $\mathrm{AgCl}_{6} \mathrm{~K}_{6}$ cluster when $\mathrm{K}^{(4)}$ ions fixed at host lattice positions (solid line) and when the six $\mathrm{K}^{(4)}$ ions are allowed to relax (dashed line). Energies are shifted by $-8180 \mathrm{eV}$.

\section{GROUND-STATE GEOMETRY}

\section{A. ROHF calculations}

In this section we first discuss the results reached within the ROHF methodology for an embedded $\mathrm{AgCl}_{6} \mathrm{~K}_{6}$ cluster where the unpaired electron is confined. In the ${ }^{2} A_{1 g}$ ground state, the unpaired electron is located in the antibonding $a_{1 g}^{*}$ orbital arising from the $5 s$ atomic orbital of free $\mathrm{Ag}^{0}$.

A first estimate of the relaxation of the $\mathrm{Cl}^{-}$ions around the $\mathrm{Ag}^{0}$ impurity is obtained using a $\mathrm{AgCl}_{6}$ cluster embedded in a similar way as described in Sec. II A. For this smaller cluster, the $6 \mathrm{~K}^{(4)}$ ions are included as AIEMP's with a $(1 s, 1 p)$ basis. In this case we only relax the first shell of $\mathrm{Cl}^{-}$ ions around the impurity and keep all other ions fixed at the lattice positions for the pure $\mathrm{KCl}$ crystal. The optimized Hartree-Fock $\mathrm{Ag}^{0}-\mathrm{Cl}$ distance for the ${ }^{2} A_{1 g}$ ground state is $3.42 \AA$, which is already $9 \%$ larger than the interatomic spacing of the $\mathrm{KCl}$ crystal. Although this relaxation is significant, one can expect that the actual $R_{e}$ value would be still larger than $3.42 \AA$ once the $\mathrm{K}^{(4)}$ ions are allowed to relax their positions. From the theory of the elasticity $R_{e}^{(4)}$ should be larger than $6.36 \AA$ if the ligand relaxation is $9 \%$.

Therefore, we optimize in a second step not only the $\mathrm{Ag}^{0}-\mathrm{Cl}^{(1)}$ distance, but also the $\mathrm{Cl}^{(1)}-\mathrm{K}^{(4)}$ distance. For this purpose we construct an $\mathrm{AgCl}_{6} \mathrm{~K}_{6}$ cluster in which the six $\mathrm{K}^{(4)}$ are now allowed to relax their position as a reaction on the outward movement of the $\mathrm{Cl}^{(1)}$ neighbors because of the presence of $\mathrm{Ag}^{0}$. Figure 3 depicts the dependence of the total energy with the $\mathrm{Ag}-\mathrm{Cl}^{(1)}$ distance comparing the cases in which the $\mathrm{K}^{(4)}$ ions are placed at the ideal lattice positions and when the $\mathrm{K}^{(4)}$ ions are at their optimized positions. It can be seen that when the six $\mathrm{K}^{(4)}$ ions are allowed to relax, $R_{e}$ becomes equal to $3.71 \AA$ and, at the same time, $R_{e}^{(4)}$ $=6.70 \AA$ for $\mathrm{Ag}-\mathrm{K}^{(4)}$ distance. By contrast, if $\mathrm{K}^{(4)}$ ions are fixed at the host lattice positions, $R_{e}=3.42 \AA$. These numbers mean (i) a total outward relaxation of almost $18 \%$ compared to the pure $\mathrm{KCl}$ crystal for the first $\mathrm{Cl}$ shell, and (ii) a significant relaxation of $6.5 \%$ for the first $\mathrm{K}^{+}$ions along $\langle 100\rangle$ directions. It is worth noting that the relaxation of lat- 
ter ions is comparable though somewhat larger than the $4.5 \%$ derived from $u=R_{e}-R_{0}=0.57 \AA$ and the theory of the elasticity. The latter theoretical model can however be considered only as a crude approximation for the present problem.

Another salient feature emerging from Fig. 3, concerns the force constant of the $A_{1 g}$ mode $k_{A}$, where only the displacement of the six $\mathrm{Cl}^{-}$ligands is involved. In fact, the resulting force constant is found to be $k_{A}=0.14$ a.u., which is five times smaller than the corresponding one derived for a $\mathrm{CrCl}_{6}{ }^{3-}$ unit. ${ }^{40}$ This force constant, which indicates that the energy minimum is rather shallow, corresponds to a vibrational frequency $\hbar \omega_{A}$ of $130 \mathrm{~cm}^{-1}$.

Let us now focus on the contribution of the $a_{1 g}^{*}$ orbital to the unpaired electron density. Within the linear combination of atomic orbitals framework the $\left|a_{1 g}^{*}\right\rangle$ molecular orbital is written as

$$
\left|a_{1 g}^{*}\right\rangle=N\left\{|5 s\rangle-\lambda_{p \sigma}\left|\chi_{p \sigma} ; \mathrm{Cl}\right\rangle-\lambda_{s}\left|\chi_{s} ; \mathrm{Cl}\right\rangle-\left|\phi_{\text {oth }}\right\rangle\right\} .
$$

Here, $|5 s\rangle$ refers to the Ag-5s atomic orbital, $\left|\chi_{p \sigma}\right\rangle$ and $\left|\chi_{s}\right\rangle$ mean symmetry adapted combinations of $3 p \sigma$ and $3 s$ orbitals of the six $\mathrm{Cl}$ ligands, while $\left|\phi_{\text {oth }}\right\rangle$ describes the contribution coming from further atoms. ${ }^{26}$ Neglecting in a first approximation the contribution of $\left|\phi_{\text {oth }}\right\rangle$ in Eq. (2), it must be verified

$$
N^{2}\left\{1+\lambda_{p \sigma}^{2}+\lambda_{s}^{2}-2\left(\lambda_{p \sigma} S_{p \sigma}+\lambda_{s} S_{s}\right)\right\}=1,
$$

where $S_{p \sigma}=\left\langle 5 s \mid \chi_{p \sigma}\right\rangle$ and $S_{s}=\left\langle 5 s \mid \chi_{s}\right\rangle$ are group overlap integrals. Condition (3) allows $N^{2}>1$ when $S_{p \sigma} \approx 0.3$ or higher. Due to the delocalization depicted in Eq. (2), the fraction of the electronic charge on the silver, called $q_{\mathrm{Ag}}$, is usually smaller than unity. Within the Mulliken approximation we have

$$
q_{\mathrm{Ag}}=f_{\mathrm{Ag}}-N^{2}\left(\lambda_{p \sigma} S_{p \sigma}+\lambda_{s} S_{s}\right),
$$

where $f_{\mathrm{Ag}}=N^{2}$ means the unpaired spin density on silver and thus is equal to $q_{\mathrm{Ag}}$ only if overlap effects are ignored. In this framework the unpaired spin densities on $3 p \sigma$ and $3 s$ orbitals of chlorine, $f_{\sigma}$ and $f_{s}$, respectively, are ${ }^{24,25}$

$$
\begin{gathered}
f_{\sigma}=\left(N \lambda_{p \sigma}\right)^{2} / 6, \\
f_{s}=\left(N \lambda_{s}\right)^{2} / 6 .
\end{gathered}
$$

At the equilibrium geometry the ROHF calculations lead to $f_{\mathrm{Ag}}=85 \%$, while the total charge on ligands is about $14 \%$ and the charge lying on further atoms is found to be smaller than $2 \%$. This high localization of the electronic charge on the $\mathrm{Ag}^{0}$ atom at the equilibrium distance turns out to be in reasonable agreement with the ratio between the experimental hyperfine constants of the ${ }^{107} \mathrm{Ag}$ isotope for the $\mathrm{KCl}: \mathrm{Ag}^{0}$ system $(A=1638 \mathrm{MHz})$ and the free-atomic isotope $\left(A_{0}\right.$ $=1718 \mathrm{MHz})^{12,14,16,17}$ The small reduction undergone by $A$ with respect to $A_{0}$ can be ascribed to the fraction of the electronic charge lying out of the silver because in a first approximation $A=f_{\mathrm{Ag}} A_{0}$.

\section{B. DFT calculations}

We now turn to the results obtained through clusters in vacuo within the DFT framework. As expected, no equilibrium distance is found for the simplest $\mathrm{AgCl}_{6}{ }^{6-}$ cluster. Ac-
TABLE I. Values (in $\AA$ ) of the equilibrium $\mathrm{Ag}-\mathrm{Cl}^{(1)}, R_{e}$, and $\mathrm{Ag}-\mathrm{K}^{(4)}, R_{e}^{(4)}$, distances obtained by means of two different DFT calculations on the 39-ions cluster. In the first calculation only the $\mathrm{Cl}^{(1)}$ ions are allowed to relax while the rest of the ions are fixed at the equilibrium distances corresponding to the perfect $\mathrm{KCl}$ lattice. In the second calculation both $\mathrm{Cl}^{(1)}$ and $\mathrm{K}^{(4)}$ ions are allowed to relax.

\begin{tabular}{ccl}
\hline \hline Calculation & $R_{e}$ & $R_{e}^{(4)}$ \\
\hline $\mathrm{K}^{(4)}$ fixed & 3.36 & 6.29 \\
$\mathrm{~K}^{(4)}$ relaxed & 3.66 & 6.70 \\
\hline \hline
\end{tabular}

cepting the Born-Mayer description, the stability of a complex requires that the involved electrostatic energy is negative. For an ionic $M X_{6}$ complex (where $X$ means a halide ion) that condition is fulfilled provided the charge of the central ion is at least equal to 2 . When the cluster size increases the total energy can, however, exhibit some minima. In the case of the simple 27-ion cluster $\mathrm{AgCl}_{6} \mathrm{~K}_{12} \mathrm{Cl}_{8}{ }^{2-}$, where only the metal ligand distance is taken as variable while the other ions are kept at their host lattice positions, the total energy shows a minimum at $3.67 \AA$. In this cluster, however, the six $\mathrm{K}^{(4)}$ ions are not considered, and the resemblance with the ROHF result is fortuitous.

A more realistic description of the $\mathrm{KCl}: \mathrm{Ag}^{0}$ system is obtained by the 39-ion cluster where up to the third neighbors of $\mathrm{Ag}^{0}$ along $\langle 100\rangle$ directions are included. As shown in Table I the equilibrium distances are quite comparable to those reached through $\mathrm{ROHF}$ calculations. In fact, we obtain $R_{e}=3.36 \AA$ when only the $\mathrm{Cl}^{(1)}$ ligands are relaxed, whereas $R_{e}$ increases to $3.66 \AA$ when the $\mathrm{Cl}^{(1)}-\mathrm{K}^{(4)}$ distance is also optimized. In this case $R_{e}^{(4)}$ is $6.70 \AA$. These findings are in good agreement with the ROHF results and also with the conclusions derived from first analysis of EPR and electronnuclear double resonance (ENDOR) data. ${ }^{24-26}$ Figure 4 depicts the calculated ground-state energy as a function of $R$ taking $R^{(4)}=6.70 \AA$. Around the minimum at $R_{e}=3.66 \AA$ a force constant of 0.15 a.u. is computed which implies $\hbar \omega_{A}$ $=137 \mathrm{~cm}^{-1}$. This value is thus again very close to the figure previously derived from ROHF methodology.

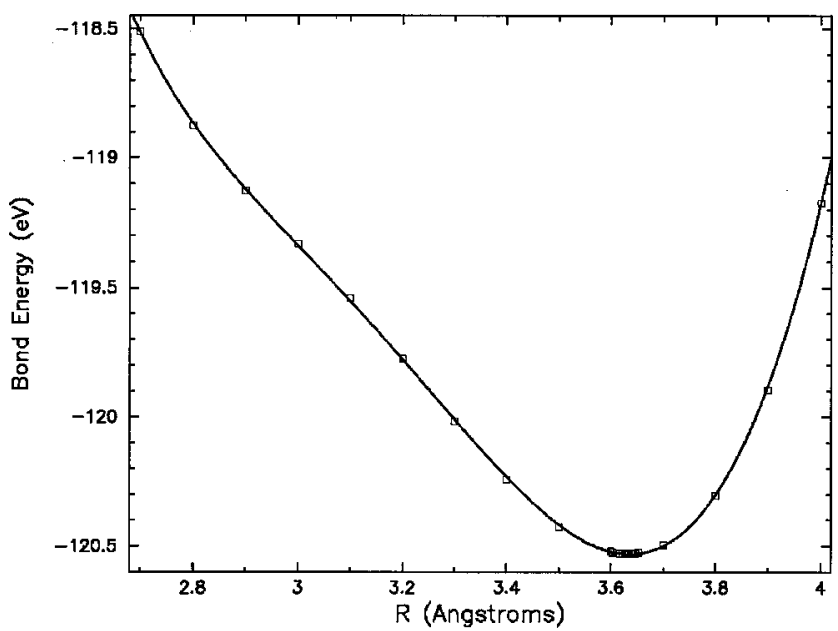

FIG. 4. $R$ (in $\AA$ ) dependence of the energy (in eV) of the 39atom cluster obtained in DFT calculations. $R_{e}^{(4)}$ is $6.70 \AA$. 
TABLE II. $R$ (in $\AA$ ) dependence of some properties obtained through DFT calculations in the 39 -atom cluster. The $\mathrm{K}^{(4)}$ ions are relaxed to $6.70 \AA$ while the rest of the atoms are placed in ideal $\mathrm{KCl}$ lattice positions. $f_{\mathrm{Ag}}, f_{s}$, and $f_{\sigma}$ represent the contribution (in $\%$ ) to the spin density of the $\mathrm{Ag}-5 s$, the $\mathrm{Cl}^{(1)}-3 s$, and the $\mathrm{Cl}^{(1)}-3 p_{\sigma}$ orbitals, respectively. $q_{\mathrm{Ag}}$ is the percentage of charge on $\mathrm{Ag}$ atom in the $a_{1 g}^{*}$ orbital.

\begin{tabular}{ccccc}
\hline \hline$R$ & $q_{\mathrm{Ag}}$ & $f_{\mathrm{Ag}}$ & $f_{s}$ & $f_{\sigma}$ \\
\hline 2.70 & 0 & 9 & 1.62 & 0.02 \\
2.80 & 0 & 8 & 2.05 & 0.02 \\
2.90 & 3 & 11 & 2.51 & 0.19 \\
3.00 & 17 & 20 & 2.89 & 0.62 \\
3.10 & 34 & 37 & 3.05 & 1.26 \\
3.20 & 51 & 60 & 2.95 & 1.97 \\
3.30 & 68 & 85 & 2.62 & 2.63 \\
3.40 & 80 & 108 & 2.16 & 3.14 \\
3.50 & 89 & 125 & 1.68 & 3.44 \\
3.60 & 93 & 135 & 1.25 & 3.55 \\
3.66 & 93 & 136 & 1.01 & 3.50 \\
3.70 & 93 & 137 & 0.90 & 3.47 \\
3.80 & 88 & 130 & 0.63 & 3.21 \\
3.90 & 81 & 120 & 0.42 & 2.88 \\
4.00 & 74 & 108 & 0.27 & 2.57 \\
\hline \hline
\end{tabular}

The unpaired spin densities derived from DFT calculations of the 39-atom cluster ground state are displayed in Table II for $2.70 \AA<R<4.00 \AA$. It turns out that for this cluster the $a_{1 g}^{*}$ charge on the silver atom $q_{\mathrm{Ag}}$ is higher than $60 \%$ only if $R>3.25 \AA$. When $R$ is around or below $3 \AA, q_{\mathrm{Ag}}$ is practically negligible. In this regime the unpaired electron is delocalized on the surface of the cluster and silver behaves as an $\mathrm{Ag}^{+}$ion. It is worth noting that for $R>3.40 \AA$ the $f_{s}$ spin density decreases significantly upon increasing $R$. In the same region, $f_{\sigma}$ is found however to be much less $R$ dependent. This behavior is in essence the same encountered in octahedral $3 d$ transition-metal complexes with halides ${ }^{3,5}$ or oxygen $^{54}$ as ligands. An explanation of these observations is given in Ref. 55. Bearing in mind the strong $R$ dependence of the $f_{s}$ spin density, the values reported in Table II can now be compared with the $f_{s}$ value of $0.88 \%$ derived from the experimental $A_{s}$ value. Such a comparison tells us that $f_{s}$ $=0.88 \%$ is scarcely compatible with $R_{e}$ around $3.30 \AA$, whereas the agreement with calculated values in the neighborhood of $R=3.70 \AA$ is certainly good.

A relevant point of the present analysis concerns the separation between the highest occupied and lowest unoccupied orbital of the cluster, and here referred to as $\Delta_{c}$. This separation models in a simple way the distance of the ground state of $\mathrm{Ag}^{0}$ center to the bottom of the conduction band of the $\mathrm{KCl}$ lattice. For the present cluster $\Delta_{c}=9000 \mathrm{~cm}^{-1}$ at $R$ $=3.25 \AA$ while $\Delta_{c}$ increases significantly to $20000 \mathrm{~cm}^{-1}$ when $R$ becomes equal to $3.80 \AA$ with $R^{(4)}$ always equal to $6.70 \AA$. As expected the degree of localization of the unpaired electron increases significantly as $\Delta_{c}$ increases.

Figure 5 and Table III display the DFT results corresponding to the 81-ion cluster. In this case a clear and deep minimum is found at $R=2.88 \AA$ as shown in Fig. 4. Such a minimum can reasonably be ascribed to an excitonlike state

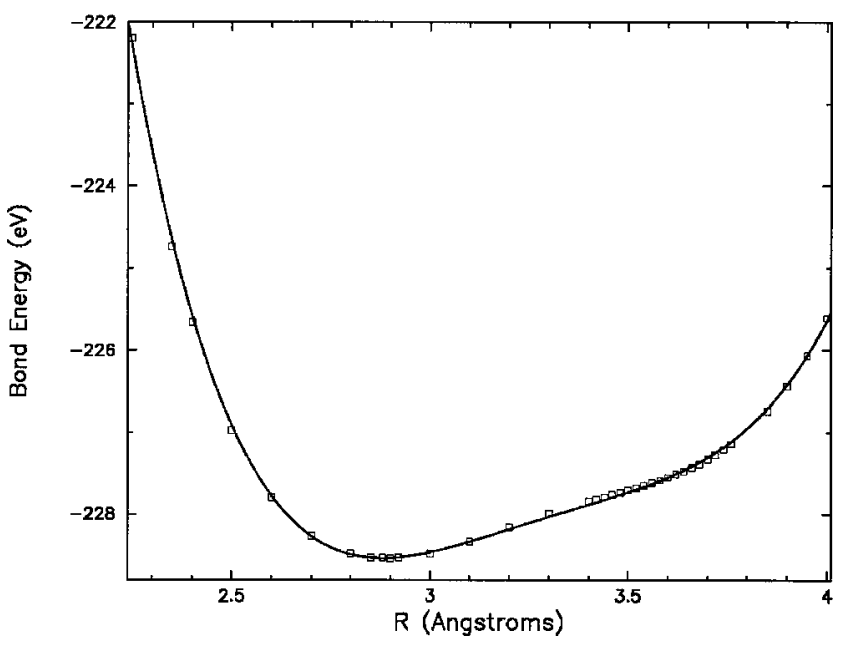

FIG. 5. $R$ (in $\AA$ ) dependence of the energy (in eV) of the 81atom cluster obtained in DFT calculations. $R_{e}^{(4)}$ is $6.70 \AA$.

consisting of an unpaired electron lying on the cluster surface and an $\mathrm{Ag}^{+}$ion at the center. The electron-hole interaction, which does not exist for the real $\mathrm{Ag}^{+}$impurity, gives rise to an artificial stabilization, masking the minimum in the $E(R)$ curve corresponding to the $\mathrm{Ag}^{0}$ impurity. This artifact is not observed in the 39-ion cluster since the surface of this cluster is much smaller and hence the kinetic energy of the unpaired electron lying on the cluster surface would be much higher. In fact the unpaired electron density on the silver atom is zero at $R=2.90 \AA$ and smaller than $10 \%$ for $R$ $<3.10 \AA$ as shown in Table III. This fact has to be related with the large $q_{K}$ values at small distances. From the behavior of impurities like $\mathrm{Mn}^{2+}, \mathrm{Ni}^{2+}$, or $\mathrm{Fe}^{3+}$ in different lattices, ${ }^{5,10,11}$ one expects a priori that $R_{e}$ for $\mathrm{KCl}: \mathrm{Ag}^{+}$is somewhat larger than $2.77 \AA$ (corresponding to $\mathrm{AgCl}$ ) and smaller than $R_{0}=3.145 \AA$ of the pure $\mathrm{KCl}$ lattice.

As regards the formation of the localized $\mathrm{Ag}^{0}$ center, no other clear minimum is found in Fig. 5 but a kind of plateau is observed for $R$ around $3.60 \AA$. Despite this fact it is worth noting that the analysis of the unpaired charge density given in Table III again supports the formation of an unpaired electron trapped at the silver atom for distances larger than $\sim 3.50 \AA$. In fact, for the 81-atom cluster the electronic charge on silver is higher than $50 \%$ only when $R>3.5 \AA$. In particular, $q_{\mathrm{Ag}}$ is close to $80 \%$ when $R$ is around $3.70 \AA$. As shown in Table III this conclusion is weakly dependent on the $R^{(4)}$ value fixed in the series. For instance the obtained $q_{\mathrm{Ag}}$ value at $R=3.60 \AA$ and the unrelaxed $R^{(4)}$ of $6.29 \AA$ is only $10 \%$ higher than that derived at the same metal-ligand distance and $R^{(4)}=6.70 \AA$.

Therefore, it can be said that ADF calculations on the cluster in vасио do not reproduce clearly the shallow minimum of the $E(R)$ curve associated with the $\mathrm{Ag}^{0}$ center stability, but at the same time they are consistent with the localization of the unpaired electron on silver for $R>3.6 \AA$. The failure of ADF calculations on a 81-atoms cluster in vacuo for well reproducing the shallow minimum, can reflect facts such as the existence of surface levels introduced artificially in the cluster in vacuo calculations or a worse description of the conduction-band states of $\mathrm{KCl}$ compared to that of ground state. Along this line it has recently been 
TABLE III. $R$ (in $\AA$ ) dependence of some properties obtained through DFT calculations in two different 81-atom clusters: unrelaxed lattice where all atoms (except $\mathrm{Cl}^{(1)}$ ) are placed in ideal $\mathrm{KCl}$ lattice positions and relaxed lattice where the $\mathrm{K}^{(4)}$ ions are relaxed to $6.70 \AA$. $f_{\mathrm{Ag}}, f_{s}$, and $f_{\sigma}$ represent the contribution (in \%) to the spin density of the $\mathrm{Ag}-5 s$, the $\mathrm{Cl}^{(1)}-3 s$, and the $\mathrm{Cl}^{(1)}-3 p_{\sigma}$ orbitals, respectively. $q \mathrm{Ag}$ and $q_{K}$ are the percentages of electronic charge on $\mathrm{Ag}$ atom and $\mathrm{K}$ (conduction band) atoms corresponding to the $a_{1 g}^{*}$ orbital.

\begin{tabular}{|c|c|c|c|c|c|c|c|c|c|c|}
\hline \multirow[b]{2}{*}{$R$} & \multicolumn{2}{|c|}{$q_{\mathrm{Ag}}$} & \multicolumn{2}{|c|}{$q_{K}$} & \multicolumn{2}{|c|}{$f_{\mathrm{Ag}}$} & \multicolumn{2}{|c|}{$f_{s}$} & \multicolumn{2}{|c|}{$f_{\sigma}$} \\
\hline & Unrelaxed & Relaxed & Unrelaxed & Relaxed & Unrelaxed & Relaxed & Unrelaxed & Relaxed & Unrelaxed & Relaxed \\
\hline 2.70 & 0 & 0 & 100 & 100 & 0 & 0 & 0.14 & 0.07 & 0.00 & 0.00 \\
\hline 2.80 & 0 & 0 & 100 & 100 & 0 & 0 & 0.18 & 0.07 & 0.00 & 0.00 \\
\hline 2.90 & 0 & 0 & 100 & 100 & 1 & 0 & 0.26 & 0.09 & 0.02 & 0.00 \\
\hline 3.00 & 1 & 0 & 99 & 100 & 2 & 1 & 0.44 & 0.18 & 0.15 & 0.03 \\
\hline 3.10 & 7 & 2 & 92 & 97 & 8 & 2 & 0.85 & 0.34 & 0.72 & 0.18 \\
\hline 3.20 & 18 & 9 & 77 & 89 & 18 & 9 & 1.25 & 0.68 & 1.71 & 0.80 \\
\hline 3.30 & 32 & 21 & 60 & 74 & 33 & 21 & 1.44 & 1.01 & 2.69 & 1.76 \\
\hline 3.40 & 46 & 35 & 44 & 57 & 49 & 37 & 1.45 & 1.18 & 3.53 & 2.68 \\
\hline 3.50 & 60 & 50 & 29 & 37 & 67 & 55 & 1.32 & 1.21 & 4.18 & 3.45 \\
\hline 3.60 & 71 & 64 & 17 & 25 & 83 & 74 & 1.08 & 1.13 & 4.61 & 4.04 \\
\hline 3.70 & 78 & 75 & 10 & 16 & 93 & 89 & 0.79 & 0.94 & 4.85 & 4.43 \\
\hline 3.80 & 82 & 84 & 5 & 13 & 99 & 98 & 0.53 & 0.84 & 4.97 & 4.60 \\
\hline
\end{tabular}

pointed out ${ }^{38}$ that fine details of the electronic structure of impurities cannot be easily obtained by means of density functional calculations on clusters. We have verified that in the present case ADF calculations are not improved by introducing the electrostatic potential due to the rest of the lattice ions lying outside the cluster, taken as point charges. A possible way for improving this situation would be the use of supercells with periodic boundary conditions.

The results obtained for the $f_{s}$ and $f_{\sigma}$ densities are quite comparable to those reached for the smaller 39-atom cluster. Again $f_{s}$ is found to be very sensitive to $R$ variations and the comparison with the $f_{s}=0.88 \%$ value (derived from the experimental $A_{s}$ ) strongly suggests that $R_{e}$ should be close to $3.7 \AA$. For this distance also the unpaired spin density on $\mathrm{Ag}$, $f_{\mathrm{Ag}}$, is in good agreement with the value of $95 \%$ derived from experiment.

Calculations performed on bigger clusters or using the simpler MS-X $\alpha$ method lead to the same conclusions. For instance, in DFT calculations on a 117-atom cluster, the behavior displayed by $q_{\mathrm{Ag}}$ and $f_{s}$ as function of $R$ is found to be similar to that for the 81-atom cluster. The shallow minimum associated with the stabilization of $\mathrm{Ag}^{0}$ is again not perfectly defined in the curve of the total energy versus $R$. In the case of MS-X $\alpha$ calculations on an 81-atom cluster the extracted $R$ dependence of $f_{s}$ is found to be very similar to that derived through DFT. For instance, in MS-X $\alpha$ calculations, $f_{s}=0.93 \%$ and $f_{\sigma}=3.07 \%$ at $R=3.70 \AA$, while at $R$ $=3.50 \AA, f_{s}$ becomes twice as large as that computed at the former distance. This result stresses that electronic properties (such as the superhyperfine tensor or the optical transitions) and its dependence on $R$ are reasonably reproduced by a simple method like MS-X $\alpha$. Unfortunately, the total-energy dependence on $R$ is not accurately reproduced mainly due to the involved muffin-tin approximation of the potential. ${ }^{56}$

In connection with the discussion above, it is worth mentioning the very recent DFT calculations carried out on $\mathrm{P}_{2} \mathrm{O}_{5}$ containing an interstitial silver atom. ${ }^{57}$ In conclusion, the presence of silver leads also to remarkable changes of the positions of bridging and nonbridging oxygens in the unit cell, although the distortion of $\mathrm{PO}_{4}$ tetrahedra is relatively small. In this case however the total charge on silver at the equilibrium geometry is equal to $+0.6 e$ reflecting the covalent character of the bond between $\mathrm{P}$ and $\mathrm{O}$ in $\mathrm{P}_{2} \mathrm{O}_{5}$.

\section{A FURTHER INSIGHT INTO Ag ${ }^{0}$ CENTERS}

\section{A. Electronic stability of the $\mathbf{A g}^{0}$ center in $\mathrm{KCl}$}

From all the preceding results it is clear that the trapping of the unpaired electron at the silver is favored by the local outward lattice relaxation around $\mathrm{Ag}^{0}$. This conclusion concurs with a previous study based on SCCEH and MS-X $\alpha$ calculations where it was pointed out that for $R<3.4 \AA$ the unpaired electron cannot be trapped at silver. ${ }^{26}$ The origin of this relevant fact can be explained by means of the following simple electrostatic model. Let us assume that the unpaired electron is highly localized on silver giving rise to an atomic $\mathrm{Ag}^{0}$ species inside $\mathrm{KCl}$. Defining $I\left(\mathrm{Ag}^{0}\right)$ as the ionization potential of free silver atom, the energy separation $\Delta_{c}$ between the bottom of the $\mathrm{KCl}$ conduction band (whose energy is $-\chi$ ) and the ${ }^{2} A_{1 g}$ ground state of the silver center can be approximated by

$$
\Delta_{c}=I\left(\mathrm{Ag}^{0}\right)-e\left\{V_{M}-V_{\text {rel }}\right\}-\chi,
$$

with $V_{M}$ the Madelung potential at a cation site of the perfect host lattice and $V_{\text {rel }}$ describes the corrections to $V_{M}$ due to the local relaxation around the silver atom. If only the ligand relaxation is taken into account, $V_{\text {rel }}$ is given by:

$$
V_{\mathrm{rel}}=6 e\left\{\frac{1}{R_{0}}-\frac{1}{R_{e}}\right\} .
$$

The contribution to $V_{\text {rel }}$ of other neighbor ions can be calculated in a similar way. For the present case, $I\left(\mathrm{Ag}^{0}\right)$ $=7.57 \mathrm{eV}, e V_{M}=8 \mathrm{eV}$, and $\chi=0.5 \mathrm{eV} .^{57}$ Therefore from Eq. (6) it comes out that $\Delta_{c}=-1 \mathrm{eV}$ if no local relaxation is taken into account. Considering the $R_{e}$ and $R_{e}^{(4)}$ values derived in Sec. III A, it is a simple matter to derive that $e V_{\text {rel }}$ is 
$3.2 \mathrm{eV}$ for the present case. Introducing these effects in Eq. (6), $\Delta_{c}$ becomes equal to $2.2 \mathrm{eV}$ and hence the ${ }^{2} A_{1 g}$ ground state of the silver center lies below the bottom of the conduction band of $\mathrm{KCl}$. In such a case the electron trapping on a localized center is stable with respect to the electronic structure of the host lattice. Notice that the value of $\Delta_{c}$ $=2.2 \mathrm{eV}$, derived from the present model, is not far from the experimental one $\left(\Delta_{c}=2.6 \mathrm{eV}\right)$ measured through photoconductivity. $^{21}$

\section{B. The origin of the force constant $\boldsymbol{k}_{A}$ in $\mathrm{KCl}: \mathbf{A g}^{\mathbf{0}}$}

In this section we focus attention on the origin of the large outward relaxation and the existence of a shallow minimum in the $E(R)$ curve. As has already been pointed out in Sec. III $\mathrm{B}$, for an isolated $\mathrm{AgCl}_{6}{ }^{6-}$ complex, the $E(R)$ curve has a repulsive profile. However, when such a complex is embedded in a crystalline lattice like $\mathrm{KCl}$ the ions close to the complex act as a kind of wall against the dissociation. In fact the dissociative tendency of the $\mathrm{AgCl}_{6}{ }^{6-}$ unit is balanced by the repulsive interaction between $\mathrm{Cl}^{(1)}$ ligands and close ions like the $\mathrm{K}^{(4)}$ placed at (100) positions. Therefore the force constant $k_{A}$ at the equilibrium geometry is expected to be comparable to that calculated from a Born-Mayer repulsive potential $U_{\mathrm{BM}}$ between a pair of $\mathrm{Cl}^{-}$and $\mathrm{K}^{+}$ions at a distance of $3 \AA$ multiplied by six. Writing $U_{\mathrm{BM}}$ as $\lambda \exp \left(-R_{14} / \rho\right)$-where $R_{14}$ is the distance between a ligand and the next $\mathrm{K}^{+}$ion-and using the standard values ${ }^{59}$ for $\lambda$ and $\rho$ of 78.5 and 0.62 a.u., it is found that $k_{A}$ equals 0.13 a.u. and $\hbar \omega_{A}=124 \mathrm{~cm}^{-1}$. These values are remarkably close to those derived in Sec. III A from ROHF calculations. This simple analysis illustrates the different behavior of the present system compared to that encountered for some other transition-metal impurities. For instance, the $A_{1 g}$ force constant of the $\mathrm{CrF}_{6}{ }^{3-}$ complex placed in fluorides can basically be understood through the force constant between $\mathrm{Cr}^{3+}$ and $\mathrm{F}^{-}$reflecting that the interaction between ligands and further neighbors in the host lattice plays a minor role. ${ }^{33} \mathrm{~A}$ similar situation is found for $\mathrm{Cr}^{4+}$ and $\mathrm{Fe}^{6+}$ in oxides. ${ }^{54}$

From the present results on the stretching $A_{1 g}$ mode, one can reasonably expect that the local frequencies of the $\mathrm{KCl}: \mathrm{Ag}^{0}$ center are relatively soft and lie below $\sim 130 \mathrm{~cm}^{-1}$. This idea is indirectly confirmed by the temperature dependence of the intensity of a phonon assisted optical transition. From such experimental data ${ }^{20}$ the frequency of a $T_{1 u}$ mode is found to be equal to $80 \mathrm{~cm}^{-1}$. Another support to this conclusion comes from the temperature dependence of the hyperfine constant $A$, which shows a linear decrease in the 77-300 $\mathrm{K}$ temperature range. ${ }^{15,17}$ In fact, as the mechanisms involving a phonon of frequency $\Omega$ includes the factor $\operatorname{coth}(\hbar \Omega / 2 K T)$ the observed temperature dependence of $A$ requires that $\Omega$ be smaller than $\sim 110 \mathrm{~cm}^{-1}$.

Other evidence, though indirect, about the proper value of $\omega_{A}$, comes from the analysis of the experimental bandwidth of the charge-transfer transition of the $\mathrm{KCl}-\mathrm{Ag}^{0}$ center. A detailed study of excited states due to $\mathrm{KCl}: \mathrm{Ag}^{0}$ is carried out in a next paper.

\section{The stability of the localized $\mathbf{A g}^{\mathbf{0}}$ center in other lattices}

From the present paper on $\mathrm{KCl}: \mathrm{Ag}^{0}$ center and the estimations of the local relaxation derived from the experimental
$A_{s}$ value, one can also try to understand the formation of a localized center in other alkali halides. The experimental $A_{s}$ constant in $\mathrm{NaCl}: \mathrm{Ag}^{0}(66.3 \mathrm{MHz})$ and $\mathrm{LiCl}: \mathrm{Ag}^{0} \quad(79.5$ $\mathrm{MHz})^{12,14,16}$ leads to $f_{s}=1.56 \%$ for the former and $f_{s}$ $=1.86 \%$ for the latter. As previously noticed ${ }^{25}$ these data indicate that $R_{e}$ decreases substantially along the host lattices series $\mathrm{KCl}-\mathrm{NaCl}-\mathrm{LiCl} . R_{e}$ has been estimated to be $\sim 3.37 \AA$ for $\mathrm{NaCl}: \mathrm{Ag}^{0}$ and $\sim 3.27 \AA$ for $\mathrm{LiCl}: \mathrm{Ag}^{0}$ implying a ligand outward relaxation of about $20 \%$ and $27 \%$ for the first and second system, respectively. ${ }^{25}$ Assuming a 7\% (9\%) relaxation of the first $\mathrm{Na}^{+}\left(\mathrm{Li}^{+}\right)$ions along $\langle 100\rangle$ directions, and taking $\chi=0.5 \mathrm{eV}, \Delta_{c}$ equals $2.2 \mathrm{eV}(3.1 \mathrm{eV})$ for $\mathrm{NaCl}: \mathrm{Ag}^{0}$ $\left(\mathrm{LiCl}: \mathrm{Ag}^{0}\right)$ using the model described in Sec. IV A. The value $\chi=0.5 \mathrm{eV}$ has been taken from experimental measurements both for $\mathrm{KCl}$ and $\mathrm{NaCl}$ whereas for $\mathrm{LiCl}$ the value of $\chi$ is not yet definitely settled. ${ }^{58}$ Therefore the increase of ligand relaxation along the $\mathrm{KCl}-\mathrm{NaCl}-\mathrm{LiCl}$ series compensates the corresponding increase of the madelung potential and the ground state of the silver center remains below the bottom of the conduction band.

A second point concerns the electronic stability of a hypothetic cationic $\mathrm{Ag}^{0}$ center in $\mathrm{AgCl}$. As $R_{0}=2.77 \AA$ is similar to the corresponding distance in $\mathrm{NaCl}$, it can tentatively be assumed that the local lattice relaxation is the same for both lattices. The main difference between alkali chlorides and $\mathrm{AgCl}$ appears, however, in the position of the bottom of the conduction band, $\chi$ being equal to $3.25 \mathrm{eV}$ for $\mathrm{AgCl}{ }^{60}$ Using the simple model described in Sec. IV A, we find $\Delta_{c}$ $=-0.5 \mathrm{eV}$ suggesting that a localized $\mathrm{Ag}^{0}$ center is not likely to be formed inside the more covalent $\mathrm{AgCl}$ lattice. Experimental results carried out on $\mathrm{Na}_{1-x} \mathrm{Ag}_{x} \mathrm{Cl}$ mixed crystals $^{27}$ reveal that such a center is formed only when the $x$ fraction is smaller than $30 \%$.

The present arguments are also helpful to clarify the actual position of silver atoms inside the $\mathrm{SrCl}_{2}$ lattice, which possess the fluorite structure. Although the formation of an $\mathrm{Ag}^{0}$ center in $\mathrm{SrCl}_{2}$ is well established by means of EPR, ${ }^{61,62}$ the associated superhyperfine structure is not fully resolved. Therefore the precise location of $\mathrm{Ag}^{0}$ in the $\mathrm{SrCl}_{2}$ lattice cell is not definitely settled. In first experiments ${ }^{61}$ it was noted that the superhyperfine structure is better observed when the magnetic field $H$ is parallel to $\langle 111\rangle$ directions than for $H$ parallel to the $\langle 100\rangle$ direction. According to this fact, it was proposed that $\mathrm{Ag}^{0}$ in $\mathrm{SrCl}_{2}$ substitutes a $\mathrm{Cl}^{-}$ion. In a subsequent work, Sekhar and Bill ${ }^{62}$ concluded however that a better agreement with EPR spectra is obtained assuming that silver replaces $\mathrm{Sr}^{2+}$. Accepting the last possibility, $e V_{M}$ $=15.6 \mathrm{eV}$, and from experimental photoemission and optical data, ${ }^{63,64} \chi$ has been estimated to lie between 0 and $1 \mathrm{eV}$. Therefore, $\Delta_{c} \approx-8 \mathrm{eV}$ in the absence of relaxation. Bearing in mind that in the series of alkali chlorides $e V_{\text {rel }}$ is estimated to be smaller than $6 \mathrm{eV}$, the formation of $\mathrm{Ag}^{0}$ at a cationic site in $\mathrm{SrCl}_{2}$ appears to be highly unlikely. This conclusion is also supported by the experimental hyperfine constant of the $\mathrm{Ag}^{0}$ center in $\mathrm{SrCl}_{2}$, which for the ${ }^{107} \mathrm{Ag}$ isotope is $\mathrm{A}$ $=1153 \mathrm{MHz} .{ }^{62}$ In fact that figure is significantly smaller than the $A$ values measured for the cationic $\mathrm{Ag}^{0}$ center in alkali halides $^{14,17}$ and involves a reduction factor of $67 \%$ with respect to the hyperfine constant of the free $\mathrm{Ag}^{0}$ atom. By contrast it is much closer to $A\left({ }^{107} \mathrm{Ag}\right)=1256 \mathrm{MHz}$ reported ${ }^{17}$ for an $\mathrm{Ag}^{0}$ atom formed at an anion site in $\mathrm{KCl}$. At 
variance with what happens for a cationic $\mathrm{Ag}^{0}$ center, the $5 s$ electron of an anionic $\mathrm{Ag}^{0}$ center experiences an attractive Madelung potential due to the rest of ions in the lattice. This favors a location of the $5 s$ level closer to the valence band of $\mathrm{KCl}$, a fact that can be responsible for the increase of the delocalization. A more detailed investigation is however necessary for explaining the main properties of the anionic $\mathrm{Ag}^{0}$ center.

\section{FINAL REMARKS AND CONCLUSIONS}

At the equilibrium geometry of the $\mathrm{KCl}: \mathrm{Ag}^{0}$ center, the present calculations stress that the unpaired electron is essentially localized on silver $(\sim 85 \%)$ and to a less extent on the ligands $(\sim 14 \%)$ in reasonable agreement with EPR data. The results embodied in Sec. III indicate that the properties of the cationic $\mathrm{Ag}^{0}$ center in $\mathrm{KCl}$ change significantly if the metalligand distance is reduced. A decrement of $R$ leads to a diminution of $\Delta_{c}$ and thus of $q_{\mathrm{Ag}}$ and $A$, favoring progressively the delocalization of the unpaired electron. From Eq. (6), it is easily derived that $\Delta_{c}$ becomes zero for $R \approx 3.35 \AA$. Assuming the bulk modulus of $\mathrm{KCl}$, this distance can be reached applying a hydrostatic pressure around $6 \mathrm{GPa}$.

The present results can also shed some light on other $s^{1}$ species (like $\mathrm{Cu}^{0}$ or $\mathrm{Cd}^{+}$) formed inside ionic lattices. For instance in the case of $\mathrm{NaCl}: \mathrm{Cu}^{0}, R_{e}$ was estimated to be $16 \%$ higher than $R_{0}{ }^{25}$ An analysis similar to that of Sec. IV $\mathrm{C}$ indicates that the ground state is located below the bottom of the conduction band of $\mathrm{NaCl}$.

As shown through this paper the knowledge of the actual equilibrium geometry for $\mathrm{KCl}: \mathrm{Ag}^{0}$ is certainly more difficult to obtain than for some transition-metal complexes with strong bonding between the central cation and ligands. For instance in the case of $\mathrm{Fe}^{6+}$ in oxides, the $\mathrm{FeO}_{4}{ }^{4-}$ complex in vacuo reproduces ${ }^{54}$ the experimental $R_{e}$ value with an error of only $3 \%$. Moreover in this complex in vacuo the force constant between the central cation and the ligands $k_{A}$ is found to be equal to 1.5 a.u. and thus only $10 \%$ smaller than the experimental one. The present results underline however that even in a difficult case like the $\mathrm{KCl}: \mathrm{Ag}^{0}$ cluster, calculations with less than 100 atoms explain to a good extent the main experimental features of the ground state.

The trapping of an unpaired electron in the conduction band of an ionic material by an $\mathrm{Ag}^{+}$ion has been shown to be closely related to a huge local relaxation around silver. Neglecting this relaxation the unpaired electron would lie above the bottom of the conduction band, as seems to be likely when $\mathrm{AgCl}$ is the host lattice. The geometrical relaxation around a substitutional atom placed in an ionic lattice has been explored through total-energy calculations of clusters centered at the impurity. The use of two different methods, and the comparison between the calculated $f_{s}$ dependence on $R$ and the $f_{s}$ value of $0.88 \%$ derived from EPR spectra of $\mathrm{KCl}: \mathrm{Ag}^{0}$ center, strongly supports that $R_{e}$ is certainly close to $3.7 \AA$.

Once the equilibrium geometry in $\mathrm{KCl}: \mathrm{Ag}^{0}$ appears to be well established and the ground-state properties reasonably understood, it is fully necessary to explore whether the rich optical spectrum ${ }^{12,19,20,17}$ can also be accounted for through the present methods. An investigation of this kind is of interest to gain a better insight into the optical properties due to $s^{1}$ and $s^{2}$ ions. ${ }^{17,65}$ Work along this line is shown in a following paper.

\section{ACKNOWLEDGMENTS}

We thank Dr. Roland Lindh of the University of Lund for valuable help with the ROHF geometry optimization calculations. The work has been financed by Spanish "Ministerio de Educación y Ciencia'" under CICyT projects PB98-1216CO2-01 and PB98-0190. Part of the computer time was provided by the "Center de Supercomputació de Catalunya," $\mathrm{C}^{4}$-CESCA, through a research grant from the University of Barcelona. C. de G. would like to thank the European Community for financial support.
*Corresponding author: Email: morenom@unican.es

${ }^{1}$ J. M. Spaeth, J. R. Niklas, and R. H. Bartram, Structural Analysis of Point Defects in Solids (Springer-Verlag, Heidelberg, 1992).

${ }^{2}$ J. H. Barkyoumb and A. N. Mansour, Phys. Rev. B 46, 8768 (1992).

${ }^{3}$ B. Villacampa, R. Alcalá, P. J. Alonso, M. Moreno, M. T. Barriuso, and J. A. Aramburu, Phys. Rev. B 49, 1039 (1994).

${ }^{4}$ I. Cabria, M. Moreno, J. A. Aramburu, M. T. Barriuso, U. Rogulis, and J. M. Spaeth, J. Phys.: Condens. Matter 10, 6481 (1998).

${ }^{5}$ M. T. Barriuso and M. Moreno, Phys. Rev. B 29, 3623 (1984).

${ }^{6}$ C. Marco de Lucas, F. Rodríguez, and M. Moreno, Phys. Rev. B 50, 2760 (1994).

${ }^{7}$ S. J. Duclos, Y. K. Vohra, and A. L. Ruoff, Phys. Rev. B 41, 5372 (1990).

${ }^{8}$ C. Marco de Lucas, F. Rodríguez, J. M. Dance, M. Moreno, and A. Tressaud, J. Lumin. 48, 553 (1991).

${ }^{9}$ A. G. Breñosa, M. Moreno, F. Rodríguez, and M. Couzi, Phys. Rev. B 44, 9859 (1991).

${ }^{10}$ B. Villacampa, R. Cases, V. M. Orera, and R. Alcalá, J. Phys. Chem. Solids 55, 263 (1994).

${ }^{11}$ J. A. Aramburu, J. I. Paredes, M. T. Barriuso, and M. Moreno, Phys. Rev. B (to be published).
${ }^{12}$ C. J. Delbecq, W. Hayes, M. C. M. O'Brien, and P. H. Yuster, Proc. R. Soc. London, Ser. A 271, 243 (1963).

${ }^{13}$ H. Seidel, Phys. Lett. 6, 150 (1963).

${ }^{14}$ P. G. Baranov, R. A. Zhitnikov, and N. I. Melnikov, Phys. Status Solidi 30, 851 (1968).

${ }^{15}$ P. G. Baranov, R. A. Zhitnikov, and N. I. Melnikov, Phys. Status Solidi 33, 463 (1969).

${ }^{16}$ G. E. Holmberg, W. P. Unruh, and R. J. Friauf, Phys. Rev. B 13, 983 (1976).

${ }^{17}$ S. V. Nistor, D. Schoemaker, and I. Ursu, Phys. Status Solidi B 185, 9 (1994).

${ }^{18}$ F. C. Brown, B. C. Cavenett, and W. Hayes, Proc. R. Soc. London, Ser. A 300, 78 (1967).

${ }^{19}$ M. Saidoh, N. Itoh, and M. Ikeya, J. Phys. Soc. Jpn. 25, 1197 (1968).

${ }^{20}$ M. Saidoh and N. Itoh, J. Phys. Soc. Jpn. 35, 1122 (1973).

${ }^{21}$ V. S. Osminin, Fiz. Tverd. Tela (Leningrad) 15, 2386 (1973) [Sov. Phys. Solid State 15, 1588 (1974)].

${ }^{22}$ M. Moreno, J. Phys. C 13, 6641 (1980).

${ }^{23}$ C. K. Jorgensen, Solid State Physics (Academic, New York, 1962), Vol. 13, p. 375; Prog. Inorg. Chem. 12, 101 (1970).

${ }^{24}$ M. Moreno, J. A. Aramburu, and M. T. Barriuso, Phys. Lett. A 87, 307 (1982). 
${ }^{25}$ M. T. Barriuso and M. Moreno, Phys. Rev. B 26, 2271 (1982).

${ }^{26}$ I. Cabria, J. A. Aramburu, M. T. Barriuso, and M. Moreno, Int. J. Quantum Chem. 61, 627 (1997).

${ }^{27}$ F. Agulló-López and F. J. López, Cryst. Lattice Defects 9, 131 (1981).

${ }^{28}$ M. Bucher, J. Phys. Chem. Solids 54, 301 (1993).

${ }^{29}$ S. Veliah, R. Pandey, and S. Marshall, Modell. Simul. Mater. Sci. Eng. 2, 933 (1994).

${ }^{30}$ R. G. Parr and W. Yang, Density-Functional Theory of Atoms and Molecules (Oxford, New York, 1989), p. 141.

${ }^{31}$ G. te Velde and E. J. Baerends, J. Comput. Phys. 99, 84 (1992).

${ }^{32}$ P. Belanzoni, E. J. Baerends, S. van Asselt, and P. B. Langewen, J. Phys. Chem. 99, 13094 (1995).

${ }^{33}$ J. A. Aramburu, M. Moreno, K. Doclo, C. Daul, and M. T. Barriuso, J. Chem. Phys. 110, 1497 (1999).

${ }^{34}$ K. Andersson, P. A. Malmqvist, B. O. Roos, A. J. Sadlej, and K. Wolinsky, J. Phys. Chem. 94, 5483 (1990).

${ }^{35}$ K. Andersson, P. A. Malmqvist, and B. O. Roos, J. Chem. Phys. 96, 1218 (1992).

${ }^{36}$ K. Andersson, M. R. A. Blomberg, M. P. Fülscher, G. Karlström, R. Lindh, P. A. Malmqvist, P. Neogrády, J. Olsen, B. O. Roos, A. J. Sadlej, M. Schütz, L. Seijo, L. Serrano-Andrés, P. E. M. Siegbahn, and P. O. Widmark, MOLCAS Version 4, University of Lund, Lund, 1997.

${ }^{37}$ K. Doclo and U. Röthlisberger, Chem. Phys. Lett. 297, 205 (1998).

${ }^{38}$ M. T. Barriuso, J. A. Aramburu, and M. Moreno, J. Phys.: Condens. Matter 11, L525 (1999).

${ }^{39}$ S. Sugano, Y. Tanabe, and H. Kamimura, Multiplets of Transition-Metal ions in Crystals (Academic Press, New York, 1970).

${ }^{40}$ K. Nakamoto, Infrared and Raman Spectra of Inorganic and Coordination Compounds, 4th ed. (Wiley, New York, 1986).

${ }^{41}$ M. T. Bennebroek, Ph.D. thesis, University of Leiden, 1996.

${ }^{42}$ M. T. Bennebroek, O. G. Poluektov, A. J. Zakrzewski, P. G. Baranov, and J. Schmidt, Phys. Rev. Lett. 74, 442 (1995).

${ }^{43}$ R. C. Baetzold and R. S. Eachus, J. Phys.: Condens. Matter 7, 3991 (1995).
${ }^{44}$ M. T. Olm, M. C. R. Symons, and R. S. Eachus, Proc. R. Soc. London, Ser. A 392, 227 (1984).

${ }^{45}$ Z. Barandiarán, L. Seijo, and S. Huzinaga, J. Chem. Phys. 93, 5843 (1990).

${ }^{46}$ Z. Barandiarán and L. Seijo, Can. J. Chem. 70, 409 (1992).

${ }^{47}$ Z. Barandiarán and L. Seijo, J. Chem. Phys. 89, 5739 (1988).

${ }^{48}$ L. Seijo and Z. Barandiarán, Computational Chemistry: Reviews of Current Trends, edited by J. Leszczynski (World Scientific, Singapore, 1999), Vol. 4, p. 55.

${ }^{49}$ L. Seijo and Z. Barandiarán, J. Chem. Phys. 94, 8158 (1991).

${ }^{50}$ J. L. Pascual, L. Seijo, and Z. Barandiarán, J. Chem. Phys. 98, 9715 (1993).

${ }^{51}$ S. H. Vosko, L. Wilk, and M. Nusair, Can. J. Phys. 58, 1200 (1980).

${ }^{52}$ A. D. Becke, Phys. Rev. A 38, 3098 (1988).

${ }^{53}$ J. P. Perdew, Phys. Rev. B 33, 8822 (1986).

${ }^{54}$ K. Wissing, J. A. Aramburu, M. T. Barriuso, and M. Moreno, J. Chem. Phys. 111, 10217 (1999).

${ }^{55}$ M. Moreno, J. A. Aramburu, and M. T. Barriuso, Phys. Rev. B 56, 14423 (1997).

${ }^{56}$ J. P. Huke, I. W. Parsons, and S. J. Till, J. Chem. Phys. 93, 1814 (1990).

${ }^{57}$ I. V. Abarenkov, I. I. Tupitsyn, V. G. Kuznetsov, and M. C. Payne, Phys. Rev. B 60, 7881 (1999).

${ }^{58}$ R. T. Poole, J. G. Jenkin, J. Liesegang, and R. C. G. Leckey, Phys. Rev. B 11, 5179 (1975).

${ }^{59}$ M. P. Tosi, Solid State Phys. 16, 1 (1964).

${ }^{60}$ J. Tejeda, N. J. Shevchik, W. Braun, A. Goldmann, and M. Cardona, Phys. Rev. B 12, 1557 (1975).

${ }^{61}$ M. Moreno, Ph.D. thesis, Universidad de Valladolid, 1973.

${ }^{62}$ Y. Ravi Sekhar and H. Bill, Phys. Status Solidi B 143, K111 (1987).

${ }^{63}$ R. T. Poole, J. Szajman, R. C. G. Leckey, J. G. Jenkin, and J. Liesegang, Phys. Rev. B 12, 5872 (1975).

${ }^{64}$ W. Hayes, Crystals with the Fluorite Structure (Clarendon, Oxford, 1974), Chap. 1.

${ }^{65}$ P. W. Jacobs, J. Phys. Chem. Solids 52, 35 (1991). 